\title{
Fast Channel Estimation Techniques for LTE Downlink Systems based on Fast Linear Toeplitz System Solver
}

\author{
Abdelhakim KHLIFI*, Ridha BOUALLEGUE* \\ * Innov'COM Laboratory, Sup'Com, University of Carthage, Tunis, Tunisia \\ abdelhakim.khlifi@gmail.com,ridha.bouallegue@ieee.org
}

\begin{abstract}
LTE is defined as the 4G generation network providing high data rates. Channel estimation is important in the wireless system conception. Classical LMMSE channel estimation techniques require $O\left(N^{3}\right)$ floating operations and $O\left(N^{2}\right)$ memory locations ( $N$ is the size of the channel autocorrelation). In this paper, we propose fast channel estimation techniques for LTE based on fast solver for linear Toeplitz system with reconstructible Cauchy-like structure. Proposed fast algorithms require only $O\left(N^{2}\right)$ floating operations and $O(N)$ memory locations. Performances of proposed fast algorithms are verified via Monte-Carlo MATLAB simulations.
\end{abstract}

Keywords- LTE; GSA; channel matrix autocorrelation; LMMSE.

\section{INTRODUCTION}

Accurate channel estimation is essential for LTE MIMOOFDM systems [2][8]. Channel estimation is based on two techniques: the pilot insertion [5][6] and the blind channel estimation [1]. The blind estimation information is based on no pilot insertion but it is not preferred for fast varying fading channel cases. The pilot-aided is based on two methods which are the block-type and the comb-type [7]. The first consists to insert the pilots into all the subcarriers of one OFDM symbol with a certain period. This technique is defined to be suitable for slow fading channels. The second technique consists to insert the pilots at some specific subcarriers in each OFDM symbol. The comb-type is preferable for fast varying fading channels and based on two steps: the channel frequency response estimation on pilot positions; by different techniques such as the Least Square (LS) and the LMMSE; then on all subcarriers by interpolation [7].

Classical LMMSE channel estimation methods, based on channel autocorrelation matrix in frequency domain [4], require $O\left(N^{3}\right)$ floating operations and $O\left(N^{2}\right)$ memory locations.

In this paper, we propose fast channel estimation techniques based on a fast solver of linear system with the application of the Generalized Schur Algorithm (GSA) which requires $O\left(N^{2}\right)$ floating operations and $O(N)$ memory locations.

The rest of this paper is organized as follows: In Section II, we introduce the LTE Downlink system model. Then we give a description of the LS, the LMMSE and the refined channel estimation technique LMMSE-DFT in Section III. In Section IV, we give a description of proposed fast channel algorithms. In section $\mathrm{V}$, simulation results are presented to demonstrate the performance of proposed techniques. Finally, section $\mathrm{V}$ concludes this paper.

\section{LTE DOWNLINK SYSTEM MODEL}

The system model is given in Fig.1. The standard LTE Downlink system is a MIMO-OFDMA based system. We consider a MIMO-OFDM system with $M_{T}$ transmit and $M_{R}$ receive antennas.

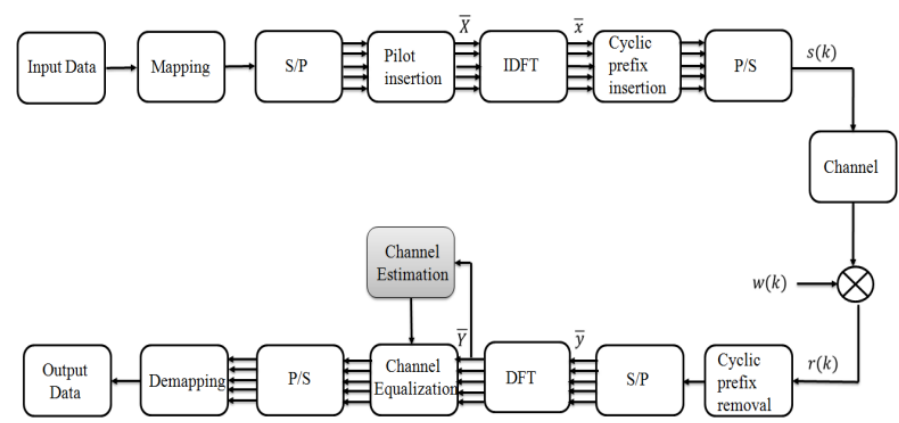

Fig. 1. System Model

LTE Downlink are OFDMA-MIMO based systems. Data streams are sent over a multi-path channel which can be modeled as a Finite Impulse Response (FIR) filter with $L$ taps for each channel path:

$$
h(t, \tau)=\sum_{l=0}^{L-1} h_{l}(t) \delta\left(t-\tau_{l}\right)
$$

We note $h_{l}$ and $\tau_{l}$ respectively the impulse response and the multipath delays of the channel. At one receive antenna, the received OFDM symbol can be written, after removing the Cyclic Prefix (CP) and performing the DFT, as: 


$$
Y=H X+\mu
$$

$X$ is the signal diagonal matrix, $H$ is the channel frequency response matrix and $Y$ is the received signals vector. The complex additive $\mu$ is the additive complex-valued white Gaussian noise vector with zero mean and variance $\sigma_{\mu}^{2}$. The transmitted signals and the noise are assumed to be independents of each other.

\section{CHANNEL ESTIMATION}

LTE systems employ Reference Signals (RSs) as pilots for channel estimation [3]. RSs are inserted in the first and the fifth OFDM symbol of the slot for extended CP or in the first and the fourth OFDM symbol for normal CP.

The received reference signals can be written as:

$$
Y_{P}=X_{P} H_{P}+\mu_{P}
$$

$(.)_{P}$ denotes positions where reference signals are transmitted.

\section{A. Least Square (LS)}

The LS estimate at the pilot subcarriers given in (3) is [6]:

$$
\begin{aligned}
\widehat{H}_{P}^{L S} & =\left(X_{P}\right)^{-1} Y_{P}=\operatorname{argmin}_{J}\left\{\left|Y_{P}-X_{P} \widehat{H}_{P}^{L S}\right|\right\} \\
& =\left[\frac{Y_{0}}{X_{0}} \frac{Y_{1}}{X_{1}} \ldots \frac{Y_{N_{P}-1}}{X_{N_{P}-1}}\right]
\end{aligned}
$$

$N_{P}$ is number of pilot symbols in one OFDM block

\section{B. Linear Mean Minimum Square Error (LMMSE)}

LMMSE channel estimation aims to minimize the mean square error between the actual and estimated value [4]:

$$
\widehat{H}_{P}^{L M M S E}=R_{H_{P} H_{P}}\left(R_{H_{P} H_{P}}+\frac{\beta}{S N R} I_{P}\right)^{-1} \widehat{H}_{P}^{L S}
$$

$R_{H_{P} H_{P}}$ represents the autocorrelation channel matrix of the subcarriers with reference signals:

$$
\begin{gathered}
R_{H_{P} H_{P}}(m, n)=E\left[H(i, m) H(i, n)^{*}\right]= \\
E\left[\sum_{k=0}^{N_{P}-1} h(i, k) \exp \left\{-\frac{j 2 \pi k m}{N_{P}}\right\} \sum_{k=0}^{N_{P}-1} h(i, k)^{*} \exp \left\{-\frac{j 2 \pi k n}{N_{P}}\right\}\right] \\
=\sum_{k=0}^{N_{P}-1} E\left[|h(i, k)|^{2} \exp \left\{-\frac{j 2 \pi k(m-n)}{N_{P}}\right\}\right] \\
=\sum_{k=0}^{L-1} \tau_{l}^{2} \exp \left\{-\frac{j 2 \pi k(m-n)}{N_{P}}\right\}
\end{gathered}
$$

We note $N_{P}$ number of pilot symbols; $\beta$ a scaling factor which depends on the signal constellation. $S N R$ is the average signal-to-noise ratio and $I_{N_{P}}$ is $N_{P} \times N_{P}$ identity matrix.

\section{C. $L M M S E-D F T$}

The inserted cyclic prefix with length of $L_{C P}$ is larger than the channel length $L$ in order to mitigate Inter Symbol Interference (IES). Therefore, the energy is concentrated in the limited paths, while only noise energy exists in the other paths. In order to reduce the noise effect, we remain only channel impulse responses within the cyclic prefix and we set the others to zeros:

$$
h_{D F T}^{L S}(k)=\left\{\begin{array}{cc}
h_{P}^{L S}(k) & \text { for } 0 \leq k \leq L_{C P}-1 \\
0 & \text { otherwise }
\end{array}\right.
$$

The refined LMMSE with DFT estimation can be written as:

$$
\widehat{H}_{P}^{L M M S E-D F T}=R_{H_{P} H_{P}}\left(R_{H_{P} H_{P}}+\frac{\beta}{S N R} I_{P}\right)^{-1} \widehat{H}_{P}^{D F T}
$$

where $\widehat{H}_{P}^{D F T}$ is the frequency domain representation of $h_{D F T}^{L S}$.

\section{PROPOSED FAST CHANNEL ESTIMATION ALGORITHMS}

LMMSE and LMMSE-DFT require the inversion of channel autocorrelation matrix. However, the inversion operation of a large dimensional matrix costs a high computational complexity.

By manipulating some mathematical operations to (6) and (8), we obtain:

$$
\left(R_{H_{P} H_{P}}+\frac{\beta}{S N R} I_{P}\right) \widehat{H}_{P}^{L M M S E}=R_{H_{P} H_{P}} \widehat{H}_{P}^{L S}
$$

$$
\left(R_{H_{P} H_{P}}+\frac{\beta}{S N R} I_{P}\right) \widehat{H}_{P}^{L M M S E-D F T}=R_{H_{P} H_{P}} \widehat{H}_{P}^{D F T}
$$

Now, in order to estimate $\widehat{H}_{P}^{L M M S E}$ or $\widehat{H}_{P}^{L M M S E-D F T}$, we are leading to solve two linear Toeplitz systems $T x=b$ and $T x_{1}=b_{1}$ where:

- $\quad T=R_{H_{P} H_{P}}+\frac{\beta}{S N R} I_{N_{P}}=\left(t_{i-j}\right)_{i, j=0}^{N_{P}-1}$ is $\quad$ a $\quad$ Toeplitz matrix.

- $\quad x=\widehat{H}_{P}^{L M M S E}$

- $x_{1}=\widehat{H}_{P}^{L M M S E-D F T}$

- $b=R_{H_{P} H_{P}} \widehat{H}_{P}^{L S}$

- $b_{1}=R_{H_{P} H_{P}} \widehat{H}_{P}^{D F T}$

$b$ and $b_{1}$ are expressed as conventional Toeplitz matrix multiplications which require both $O\left(N_{P}{ }^{2}\right)$ operations. They can be fast computed by FFT transform requiring then $O\left(N_{P} \log N_{P}\right)$ operations [11].

$T \in \mathbb{C}^{N_{P} \times N_{P}}$ satisfies the Sylvester displacement [9]:

$$
E T-T Z=G V^{H}
$$


where $G, V \in \mathbb{C}^{N_{P} \times r}$ are full rank matrices, called the generators, $E, Z \in \mathbb{C}^{N_{P} \times N_{P}}$ are the displacement matrices and $r$ is the displacement rank. In the Toeplitz case, $r=2$.

$$
\begin{aligned}
& E=\left[\begin{array}{cc}
0 & 1 \\
I_{N_{P}}-1 & 0
\end{array}\right] \\
& Z=\left[\begin{array}{cr}
0 & -1 \\
I_{N_{P}}-1 & 0
\end{array}\right] \\
& G=\left[\begin{array}{cccc}
1 & 0 & \ldots & 0 \\
t_{0} & t_{1-N_{P}}+t_{1} & \ldots & t_{-1}+t_{N_{P}-1}
\end{array}\right]^{T}
\end{aligned}
$$

$V$

$=\left[\begin{array}{cccc}t_{N_{P}-1}-t_{-1} & \ldots & t_{1}-t_{1-N_{P}} & t_{0} \\ t_{0} & t_{1-N_{P}}+t_{1} & \ldots & t_{-1}+t_{N_{P}-1}\end{array}\right]$

The Toeplitz matrix $T$ can be transformed to a Cauchy-like matrix [10]:

We pose:

$$
C=F_{1}^{H} T F_{-1}
$$

- $\quad w_{N_{P}}^{+}(k)=e^{\frac{i \pi}{N_{P}}(2 k)}$ and $w_{N_{P}}^{-}(k)=e^{\frac{i \pi}{N_{P}}(2 k+1)}$ respectively the $N_{P}$-roots of 1 and -1 .

- $\quad F_{1}$ and $F_{-1}$ are respectively the FFT and IFFT operations.

$$
\begin{aligned}
F_{1} & =\frac{1}{\sqrt{N_{P}}}\left[\left(w_{N_{P}}^{+}(l)\right)^{k}\right]_{l, k=0,1, \ldots, N_{P}-1} \\
F_{-1} & =\frac{1}{\sqrt{N_{P}}}\left[\left(w_{N_{P}}^{-}(l)\right)^{k}\right]_{l, k=0,1, \ldots, N_{P}-1}
\end{aligned}
$$

We define:

$$
K=\left(k_{0}, k_{1}, \ldots, k_{N_{P}-1}\right) \text { and } M=\left(m_{0}, m_{1}, \ldots, m_{N_{P}-1}\right)
$$

Then (13) becomes:

$$
K C-C M=\tilde{G} \tilde{V}^{H}
$$

where

$$
\begin{gathered}
E=F_{1} M F_{1}^{H} \\
Z=F_{-1} K F_{-1}^{H} \\
\tilde{G}=F_{1} G \\
\tilde{V}=F_{-1} V
\end{gathered}
$$

We are leading now to solve $C \tilde{x}=B$ where $\tilde{x}=F_{-1} x$ and $B=F_{1} \mathrm{~b}$.

In [12], a fast solver for linear systems with displacement structure by the application of the GSA has been proposed.

The GSA computes the factorization of $C$ :

$$
C=\left[\begin{array}{cc}
d & u^{H} \\
l & \tilde{C}
\end{array}\right]=\left[\begin{array}{cc}
1 & 0 \\
\frac{1}{d} l & I_{N_{P}-1}
\end{array}\right]\left[\begin{array}{cc}
d & u^{H} \\
0 & S_{1}(C)
\end{array}\right]
$$

$S_{1}(C)=\tilde{C}-\frac{1}{d} l u^{H}$ is the first Schur complement of $C$.

We associate to the Cauchy-like system the augmented matrix $A_{C, B}$ :

$$
A_{C, B}=\left[\begin{array}{cc}
C & B \\
-I_{N_{P}} & O
\end{array}\right]_{\left(2 N_{P}\right) \times\left(N_{P}+1\right)}
$$

Now, in order to estimate $\widehat{H}_{P}^{L M M S E}$ or $\widehat{H}_{P}^{L M M S E-D F T}$, we are leading to compute $S_{N_{P}}\left(A_{C, B}\right)$ by applying $N_{P}$ steps of the GSA to $A_{C, B}$.

Matlab notation for component-wise division (./) and for subindexing (:) are used in the algorithm.

For example, if $a$ and $e$ are vectors of size $(n \times 1)$ and $A=\left(a_{i, j}\right)$ an $(m \times n)$ matrix, then:

$$
\begin{gathered}
a . / e=\left(a_{1} / e_{1}, a_{2} / e_{2}, \ldots, a_{n} / e_{n}\right)^{T} \\
A_{2: 8,:}=\left(a_{i, j}\right), \quad i=1, \ldots, 8 \text { and } j=1, \ldots, n
\end{gathered}
$$

The proposed method computes $\tilde{G}, \tilde{V}, K, M$ and $B$ with the cost of $\boldsymbol{O}\left(N_{P} \log \left(N_{P}\right)\right)$ operations and applies then the GSA in $\boldsymbol{O}\left(N_{P}{ }^{2}\right)$ operations. In addition, the proposed algorithm requires only the computation of the first row and the first column of $R_{H_{P} H_{P}}$ instead of the computation of all matrix components for classical LMMSE algorithms.

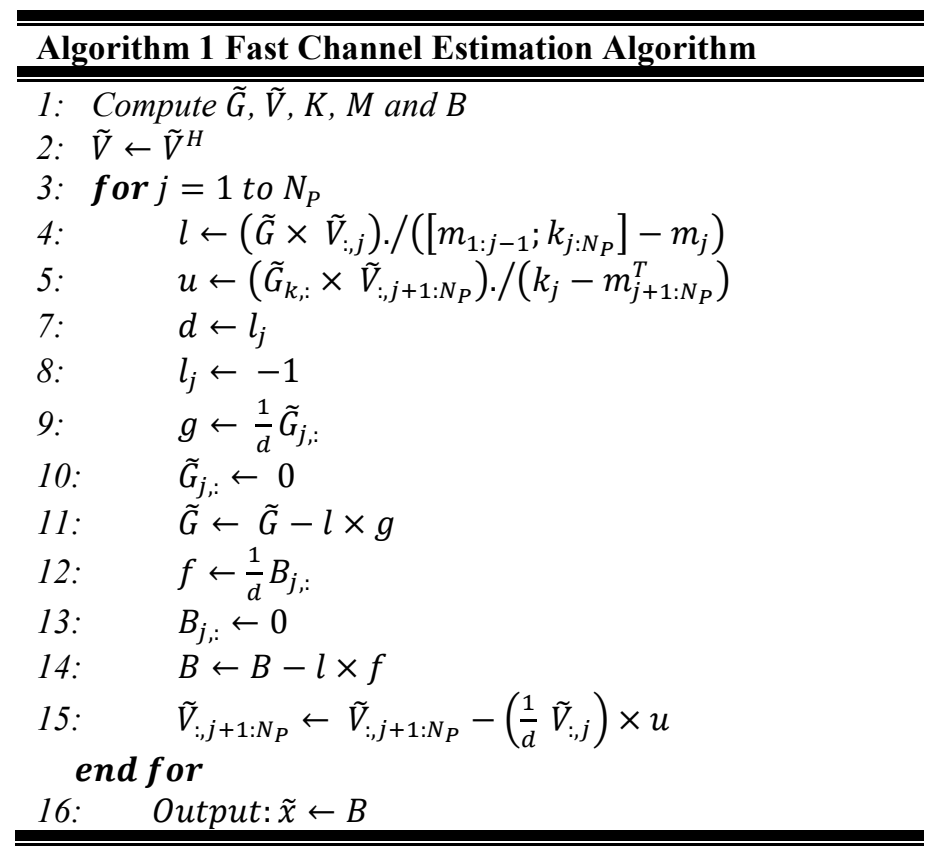

After computing the Cauchy system solution, we have to recover the Toeplitz solution:

$$
\widehat{H}_{P}^{L M M S E}=F_{-1}^{H} \tilde{x}
$$


In the same way, to get the fast LMMSE-DFT channel estimator, we apply the same algorithm while changing $B$ by $B_{1}=F_{1} b_{1}$.

\section{Simulations Results}

We investigate the performance proposed fast channel estimation techniques for LTE-20MHz system. Table 1 gives a summary of used simulation parameters.

TABLE I. SIMULATION PARAMETERS

\begin{tabular}{l|c}
\hline LTE system & $20 \mathrm{MHz}$ \\
\hline FFT size & 2048 \\
\hline Number of used subcarriers & 1200 \\
\hline CP length & 512 \\
\hline $\begin{array}{l}\text { Number of OFDM symbols } \\
\text { per Time Slot }\end{array}$ & 14 \\
\hline Number of LTE frames & 10 \\
\hline Number of pilots & 200 \\
\hline Channel Type & Rayleigh \\
\hline MIMO & $2 \times 2$ \\
\hline Modulation & QPSK \\
\hline
\end{tabular}

The number of pilots is 200 in one OFDM block. In this case, the channel matrix autocorrelation has a size of $200 \times$ 200. The proposed algorithm computes the generator matrices $\tilde{G}$ and $\tilde{V}$; the displacement matrices ; $K$ and $M$; and $B$ with the cost of $\boldsymbol{O}(200 \log (200)) \approx \boldsymbol{O}(400)$ operations and applies then the GSA in $\boldsymbol{O}\left(200^{2}\right)$ operations. In total, the proposed algorithm requires $\boldsymbol{O}(400)+\boldsymbol{O}\left(200^{2}\right)$ operations.

These analytical results are also verified via computer-based Monte-Carlo simulation results. Simulation results show that fast LMMSE techniques has the same performances as well as the classical LMMSE techniques in terms of BER and MSE as shown in Fig.2 and Fig.3 which are both better than LS estimator.

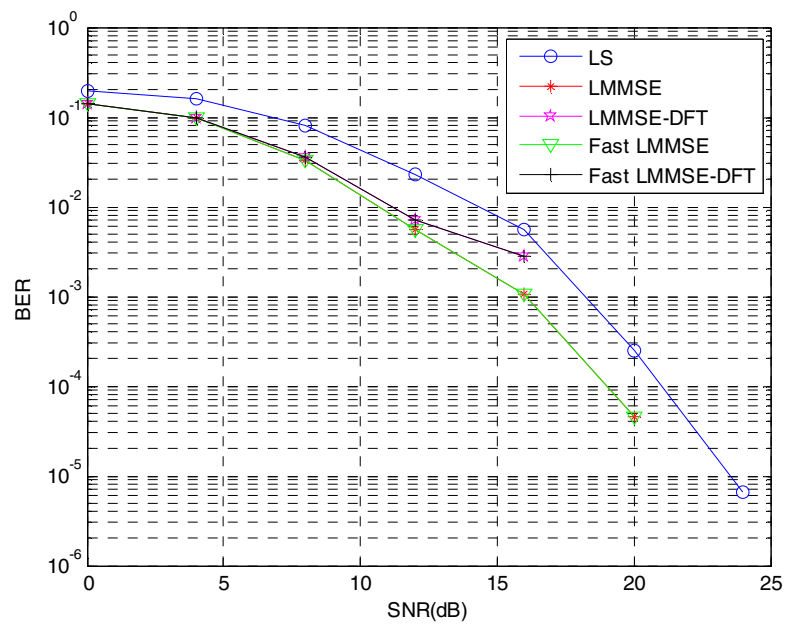

Fig. 2. BER versus SNR

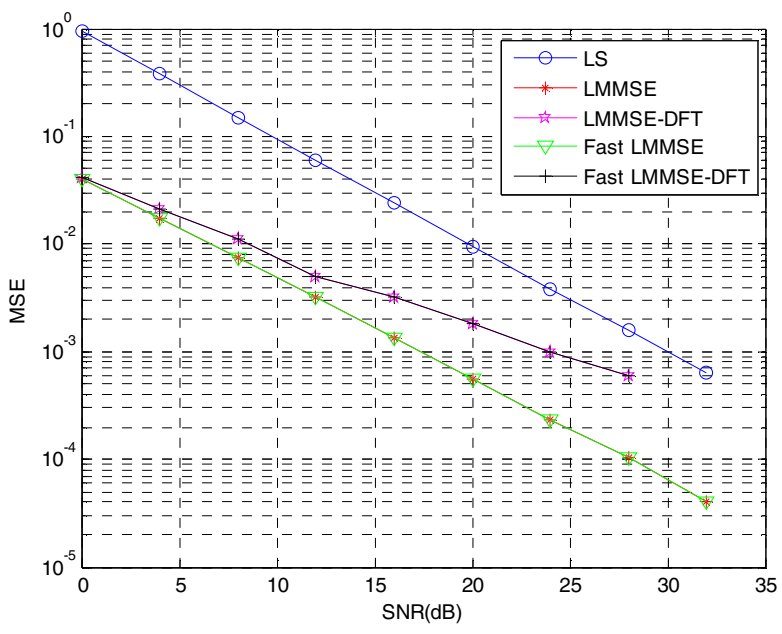

Fig.3. MSE versus SNR

\section{CONCLUSION}

Fast LMMSE and LMMSE-DFT algorithms have been proposed for LTE downlink systems based on a fast linear Toeplitz system solver. Traditional LMMSE algorithms need an inversion operation of the autocorrelation matrix which require $O\left(N^{3}\right)$ operations and $O\left(N^{2}\right)$ memory locations $(N$ is the size of the autocorrelation matrix). For a large dimensional matrix, the inversion operation assumes a high computational complexity. Therefore, and based on the structure of the autocorrelation matrix, we have proposed fast channel estimation techniques based on a linear Toeplitz solver by the application of the Generalized Schur Algorithm which requires only $O\left(N^{2}\right)$ operations and $O(N)$ memory locations.

\section{REFERENCES}

[1] R. Lin and A. P. Petropulu, "Linear precoding assisted blind channel estimation for OFDM systems," IEEE Transactions on Vehicular Technology, vol. 54, no. 3, pp. 983-995, 2005.

[2] D. Wan, B. Han, J. Zhao, X. Gao, and X. You, "Channel estimation algorithms for broadband MIMO-OFDM sparse channel," Proc.14th IEEE Int. Symp. on Personal, Indoor and Mobile Radio Communications, pp.1929-1933, Beijing, China, Sept. 2003.

[3] 3GPP, "Evolved Universal Terrestrial Radio Access (E-UTRA); Physical channels and modulation," TS 36.211, 3rd Generation Partnership Project (3GPP), Sept. 2008.

[4] J.-J. van de Beek, O. Edfors, M. Sandell, S. K. Wilson, and P. O. Borjesson, "On channel estimation in OFDM systems," in Proc. IEEE 45th Vehicular Technology Conf., Chicago, IL, Jul. 1995, pp. 815-819.

[5] O. Edfors, M. Sandell, J.-J. van de Beek, S. K. Wilson and P. O. Borjesson, "OFDM channel estimation by singular value decomposition,"in Proc. IEEE 46th Vehicular Technology Conference, Atlanta, GA, USA,Apr. 1996, pp. 923-927.

[6] J.-C. Lin, "Least-Squares Channel Estimation for Mobile OFDM Communication on Time-Varying Frequency-Selective Fading Channels, IEEE Transactions on Vehicular Technology, vol.57, no.6, pp.35383350, 2008.

[7] M.-H. Hseich and C. - H. Wei "Channel estimation for OFDM systems based on comb-type pilot arrangement in frequency selective fading 
channels," IEEE Transactions on Consumer Electronics, vol. 44, no. 1, pp. 217-225, 1998.

[8] M. Simko, D. Wu, C. Mehlführer, J. Eilert and D. Liu, "Implementation Aspects of Channel Estimation for 3GPP LTE Terminals" in Proc. Proc. European Wireless 2011, Vienna, April, 2011.

[9] G. Heinig and K. Rost. Algebraic Methods for Toeplitz-Like Matrices and Operators, volume 13 of Operator Theory: Advances and Applications. Birkh"auser Verlag, Basel, 1984.

[10] G. Heinig and A. Bojanczyk. Transformation techniques for Toeplitz and Toeplitz-plus-Hankel matrices. I. Transformations. Linear Algebra Appl.,254:193-226, 1997.

[11] C. Van Loan, Computational Frameworks for the Fast Fourier Transforms, Frontiers Appl.Math. 10, SIAM, Philadelphia, 1992.

[12] A. Arico and G. Rodriguez. A fast solver of linear systems with displacement strucutre. Springer Numerical Algorithms, vol. 55, no. 4, pp. 529-556, Dec.2002.

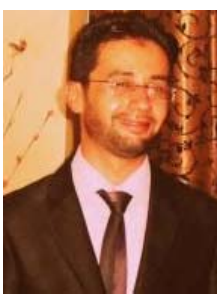

Abdelhakim Khlifi was born in Düsseldorf, Germany, on January 07,1984 . He graduated in Telecommunications Engineering, from National Engineering School of Gabès in Tunisia, July 2007.In June 2009, he received the master's degree of research in communication systems of the School of Engineering of Tunis ENIT. Currently, he is a Ph.D student at the National School of Engineering of Tunis. His research spans radio channel estimation in LTE MIMO-OFDM systems.

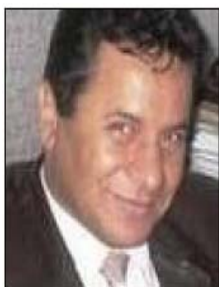

Ridha Bouallegue was born in Tunis, Tunisia. $\mathrm{He}$ received the M.S degree in Telecommunications in 1990 the Ph.D. degree in Telecommunications in 1994, and the Habilitation a Diriger des Recherches (HDR) degree in Telecommunications in 2003, all from the National Engineer School of Tunis (ENIT), Tunisia. He is currently Professor in the National Engineer School of Tunis (ENIT) and Director of Research Laboratory Innov'COM Sup'Com. His current research interests include mobile and satellite communications, Access technique, intelligent signal processing, CDMA, MIMO-OFDM and UWB system. 